Conclusion In 138 colonoscopy procedures performed in a large Trust, $21 \%$ did not adhere to the minimal withdrawal time of $6 \mathrm{~min}$ as recommended in the best practice guidelines. If this finding were replicated across the UK, a significant amount of pathology may be missed, increasing risks to our patients.

\section{PTH-046 DOUBLE-BALLOON ENTEROSCOPY ASSISTED CYANOACRYLATE INJECTION THERAPY OF SMALL- BOWEL VARICES: INTERNATIONAL EXPERIENCE FROM TWO EUROPEAN CENTRES}

\begin{abstract}
${ }^{1}$ Alberto Murino*, ${ }^{2}$ Andrea May, ${ }^{1}$ Nikolaos Lazaridis, ${ }^{1}$ Nikolaos Koukias, ${ }^{1}$ Erasmia Vlachou, ${ }^{3}$ Katie Planche, 'David Patch, 'Edward I Despott. 'Royal Free Unit For Endoscopy, The Royal Free Hospital and University College London (UCL), Institute For Liver And Digestive Health, Hampstead, London, London, UK; ${ }^{2}$, Department of Gastroenterology, Sana Klinikum Offenbach GmbH, Offenbach, Germany; ${ }^{3}$, Department of Radiology, The Royal Free Hospital and University College London (UCL) Institute for Liver and Digestive Health, Hampstead, London, London, UK
\end{abstract}

\subsection{6/gutjnl-2018-BSGAbstracts.67}

Introduction Small bowel varices (SBV) are a rare consequence of portal hypertension and could lead to life-threatening midgut bleeding. Radiological intervention (RI) is usually considered first line management (e.g. Trans-jugular intrahepatic portosystemic shunting (TIPS), stenting of occluded mesenteric veins \pm embolisation of culprit varices). In cases where $\mathrm{RI}$ is impossible, management options become very limited.

This multicentre case series evaluated the usefulness of double-balloon enteroscopy (DBE) assisted cyanoacrylate injection of SBV.

Methods Retrospective review of DBE facilitated cyanoacrylate injection of SBV (December 2015 to October 2016). Demographic, clinical, endoscopic and radiological findings, interventions and follow-up data were analysed.

Results Ten DBEs were performed in 6 patients (4 women, median age: 68.5 years). Five patients had previous surgery (hemi-hepatectomy $(n=2)$; SB resection $(n=2)$; appendicitis with peritonitis $(n=1))$; one patient had a history of intraabdominal sepsis in childhood causing portal vein thrombosis and one had cryptogenic thrombosis of the portal and the mesenteric vein. No radiological or surgical options were deemed feasible in any case. SBV were diagnosed at capsule endoscopy and triple phase CT mesenteric angiography. At DBE, a total of 13 nests of SBV were identified and injected with cyanoacrylate glue. There were no haemorrhagic or embolic complications but 1 patient developed an infection of a congenital urachal cyst, which was treated successfully with antibiotics. All patients underwent DBEs via the anterograde route, 2 patients required bi-directional DBE for treatment of both proximal and distal SBV and in total 2 patients required a repeat DBE for further treatment of SBV. At 30 day followup post-therapy, only 1 patient had experienced a mild recurrence of mid-gut bleeding treated conservatively. One patient presented with acute gastrointestinal bleeding 7 months later and a repeat DBE with cyanoacrylate injection therapy was successfully performed. One patient was lost to follow-up. The remaining patients had 12 months of follow-up without any recurrent gastrointestinal bleeding.

Conclusion Cyanoacrylate injection therapy of SBV at DBE appears to be a safe and effective management strategy for this condition when other first-line options are not feasible.

\section{PTH-047 MICROSCOPIC COLITIS IN PATIENTS UNDERGOING TWO WEEK WAIT COLONOSCOPY FOR CHRONIC DIARRHOEA}

Jeremy Nayagam*, Sarah Fleming, Vishal Mehta, Alistair McNair, Aathavan Loganayagam. Queen Elizabeth Hospital, Lewisham and Greenwich NHS Trust, UK

\subsection{6/gutjnl-2018-BSGAbstracts.68}

Introduction Chronic diarrhoea is an indication for referral on the Colorectal two week wait (TWW) pathway for suspected malignancy. Microscopic colitis (MC) characteristically presents with chronic watery diarrhoea. The frequency of MC diagnosed in TWW patients has not been systematically evaluated, but from available literature is diagnosed in $0.1 \%-2.8 \%$ of patients. We sought to define the incidence of $\mathrm{MC}$ in our cohort of patients who underwent colonoscopy for chronic diarrhoea on TWW pathway.

Methods All colonoscopies performed in a single NHS District General Hospital over a 4 year period (1/1/12 - 31/12/15) under the TWW pathway with one of the indications for investigation listed as chronic diarrhoea were identified from the electronic endoscopy database. Information on patient demographics, endoscopy reports and histological diagnoses were collected. For patients with histology definitive or suspicious for $\mathrm{MC}$, the slides were re-reviewed by a single Histopathology Consultant with an interest in gastrointestinal disease. Further clinical information from case notes was obtained for definitive cases of MC.

Results Colonoscopy was performed in 533 patients in this time period, with a median age of 62 years, $55.2 \%$ were female. Out of these, $91.2 \%$ had colonic biopsies taken, and $84.2 \%$ had colonic biopsies from macroscopically normal mucosa, to exclude the diagnosis of MC. 21 patients $(3.9 \%$ of all patients) were diagnosed with MC (6 with collagenous colitis, 15 with lymphocytic colitis). This subgroup had a median age of 76 years, $76.2 \%$ were female. $48 \%$ were on proton pump inhibitors, $19 \%$ had a diagnosis of hypothyroidism, $10 \%$ had a known malignancy (non colorectal).

Conclusions In our experience, random colonic biopsies were taken in the majority of patients referred on the TWW pathway with chronic diarrhoea in order to assess for MC. MC is a relatively common diagnosis and from our cohort of patients it was mainly seen in elderly females. MC should be considered in patients with chronic diarrhoea undergoing colonoscopy for suspected malignancy.

\section{PTH-048 DIAGNOSTIC ERCP IN SPECIALIST CENTRES: SAFE AND EFFECTIVE FOR EVALUATING INDETERMINATE BILIARY CHANGES FOLLOWING MDT}

Wei On*, Laura Dwyer, Rizwan Saleem, Steve Hood, Richard Sturgess, Nick Stern. University Hospital Aintree, Liverpool, UK

\subsection{6/gutjnl-2018-BSGAbstracts.69}

Introduction Indeterminate biliary strictures (BS) may be identified in symptomatic patients or incidentally on imaging. Patients need investigation to exclude cholangiocarcinoma or other significant pathology. The first line investigation is with Magnetic Resonance Cholangio-Pancreatography (MRCP) however this is not always diagnostic. Such cases are all reviewed 